\title{
Electrokinetic and magnetic fields generated by flow through a fractured zone: a sensitivity study for La Fournaise volcano
}

\author{
Pierre M. Adler, Jean-Louis Le Mouel \\ Institut de Physique du Globe de Paris, Paris, France
}

Jacques Zlotnicki

Laboratoire de Géophysique d'Orléans, CNRS, Orléans, France

\begin{abstract}
A number of electric and magnetic signals have been observed on La Fournaise volcano, attributed to electrokinetic effects. A simple model is proposed here to check this hypothesis. The volcano is idealized as a $2 \mathrm{~d}$ heterogeneous structure composed of a fractured zone located between two porous zones through which meteoritic waters flow downwards; flow occurs predominantly in the fractured zone and induces electromagnetic fields. Correct orders of magnitude are obtained for the measured surface fields. The importance of the heterogeneous character of the underground medium is demonstrated; local measurements of various quantities are recommended.
\end{abstract}

\section{Introduction}

It has been proposed that anomalous electric and magnetic fields observed before earthquakes and volcanic activity could be generated by the electrokinetic effect induced by water flow resulting from internal stresses, thermal buoyancy effects and meteoritic waters. The order of magnitude and the sign of the magnetic field were correctly obtained for the Matsushiro earthquake swarm [Mizutani and Ishido, 1976]. Self-potentials and magnetic measurements at La Fournaise volcano have been analyzed and interpreted in the same way [Zlotnicki and Le Mouel, 1990; Michel and Zlotnicki, 1998].

These two series of large-scale observations were substantiated by local measurements made on plugs in order to obtain dzeta-potentials and streaming potential coefficients of various minerals and rocks as functions of physico-chemical parameters such as salt concentration, ph and temperature [Ishido and Mrzutani, 1981; Martin et al., 1982; Morgan et al., 1989; Antraygues and Aubert, 1993; Hovhannissian, 1999; Lorne et al., 1998]. These data confirmed that the eletrokinetic effect could generate the observed large-scale fields, and is more plausible for volcanoes than previously suggested thermo- and piezo-magnetism mechanisms.

However, there has been little quantitative modeling of a precise physical situation, although Fitterman [1978,1979] analyzed the surface electric potential anomaly induced by a buried pressure source in a layered half-space and the surface

Copyright 1999 by the American Geophysical Union.

Paper number 1999GL900095.

0094-8276/99/1999GL900095\$05.00 magnetic field in the vicinity of a vertical fault ( $c f$ also the recent review by Johnston, 1997).

The major purpose of the present note is to provide a sensitivity analysis of the electrokinetic effect for La Fournaise volcano. This paper is organized as follows. An idealized model of the system is proposed and the governing equations are presented as well as the method of resolution and the range of parameters. The last section is devoted to a presentation and discussion of the results.

\section{General}

La Fournaise volcano (Réunion Island), $2630 \mathrm{~m}$ high, is one of the most active basaltic volcanoes in the world with one eruption every 18 months. It is characterized by heavy rainfalls reaching $6 \mathrm{~m}$ per year and large anomalies in the electric potential, which may be of the order of $2 \mathrm{~V}$ [M $\mathrm{Mchel}$ and Zlotnickn, 1998]. A large fracture zone, about $500 \mathrm{~m}$ wide, cuts across the whole volcano. As mentionned by Zlotnick and Le Mouel, [1990], this zone is likely to play an important part in the water circulation through the massif.

For this study, we adopt the simplified structure shown in Figure 1 which presents a cross-section of the volcano perpendicular to the fracture zone; the topography is simplified so that the cross-section is of a constant height $L \sim 2$ $\mathrm{km}$, of the order of the height of the volcano; the top corresponds roughly to the top of the volcano and the bottom to sea level. This cross-section consists of three zones; the two external zones are assumed to be a porous medium with identical properties surrounding a fractured zone of width $2 h \approx 500 \mathrm{~m}$ with different properties. For simplicity, the structure is assumed to be translationnally invariant along the $z$-axis which is perpendicular to the plane of the figure.

Meteoritic water is assumed to flow through this structure. The upper and lower boundaries of the porous medium ( $x=0$ and $L,|y| \geq h$ ) are assumed to be impermeable; hence, water flows through the central zone and drags along water contained in the porous medium. We investigate the influence of this flow on the electric potential and the magnetic field which can be measured at the volcano surface $(x=0)$.

When an electrolyte flows through a charged medium, electric and convective phenomena are coupled [de Groot and Mazur, 1969; Nourbehecht, 1963]. The seepage velocity $\boldsymbol{u}$ and the current density $\boldsymbol{I}$ are linearly related to the driving forces which are the gradients of the pressure $p$ and of the 


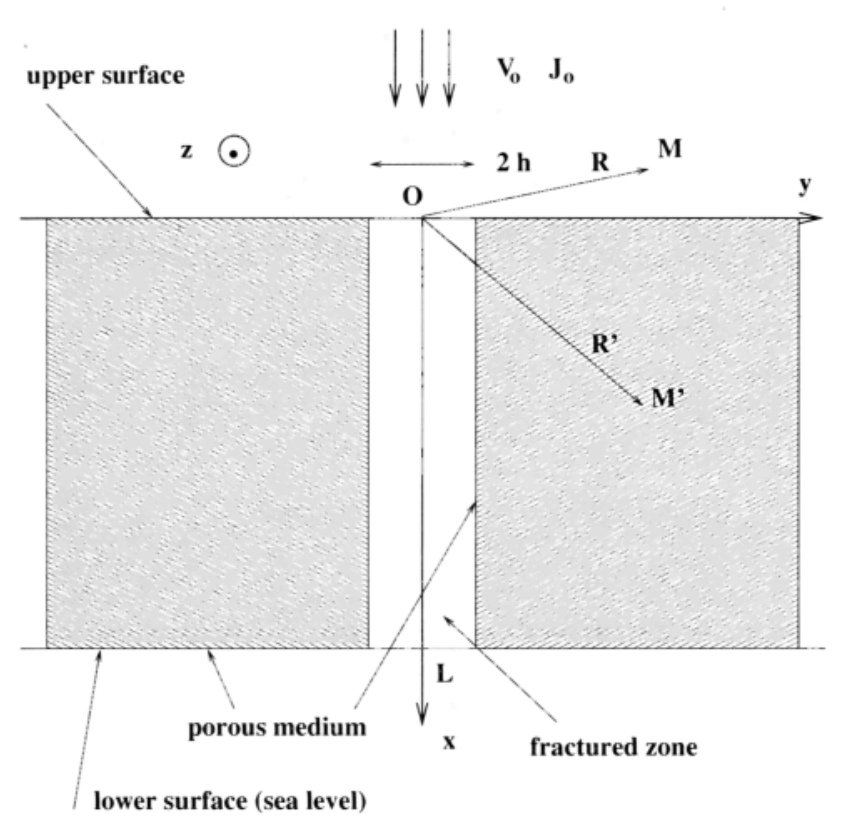

Figure 1. Model schematic of La Fournaise volcano. $V_{0}$ and $J_{0}$ are defined in (5). The $x$ - and $y$-axes are vertical and horizontal, respectively.

electric potential $\psi$

$$
\begin{aligned}
& \boldsymbol{I}_{\boldsymbol{\imath}}=-\sigma_{2} \nabla \psi_{i}-\alpha_{2} \nabla p_{i} \\
& \boldsymbol{u}_{\boldsymbol{2}}=-\alpha_{i} \nabla \psi_{i}-\frac{K_{i}}{\mu} \nabla p_{i}
\end{aligned}
$$

where the subscript $i$ stands for the porous medium $(i=p)$ or for the fractured medium $(i=f), \sigma_{i}$ is the electrical conductivity of the medium $i, K_{i}$ its permeability and $\alpha_{i}$ the electroosmotic coupling coefficient. $\mu$ is the viscosity of the fluid.

Conservation of mass and current requires that $\boldsymbol{u}_{i}$ and $I_{i}$ satisfy the continuity equations

$$
\nabla \cdot I_{\imath}=0, \nabla \cdot u_{i}=0, \quad \text { for } i=p, f
$$

This system has to be supplemented by boundary conditions. At $|y|=h, x \geq 0$, pressure, potential and normal fluxes are all continuous,

$$
p_{p}=p_{f}, \psi_{p}=\psi_{f}, \boldsymbol{n} \cdot \boldsymbol{I}_{p}=\boldsymbol{n} \cdot \boldsymbol{I}_{f}, \boldsymbol{n} \cdot \boldsymbol{u}_{p}=\boldsymbol{n} \cdot \boldsymbol{u}_{f}
$$

where $n$ denotes the unit normal to the discontinuity.

At $x=0$ and $L$, for $|y| \geq h$, no flux crosses the'surface. For $|y| \leq h$, the local seepage velocity $V_{0}$ and local current density $J_{0}$ are assumed not to vary with $y$. To summarize,

$$
\begin{aligned}
& |y| \geq h, x=0 \quad \text { and } L: u_{p x}=0, \quad I_{p x}=0 \\
& |y| \leq h, x=0 \quad \text { and } L: u_{f x}=V_{0}, I_{f x}=J_{0}
\end{aligned}
$$

Other boundary conditions could have been chosen, such as $\psi=$ constant at $x=L$; however, measurements are performed at $x \sim 0$, and are insensitive to the conditions at $x=L$.

\section{Solution and range of parameters}

Because of the symmetry of the model with respect to the $x$-axis, the general solution for $y>0$ can be written as

$$
\begin{aligned}
& p_{p}=\sum_{n=0}^{\infty} a_{n} \cos \left(\frac{n \pi x}{L}\right) \exp \left(-\frac{n \pi y}{L}\right) \\
& \psi_{p}=\sum_{n=0}^{\infty} c_{n} \cos \left(\frac{n \pi x}{L}\right) \exp \left(-\frac{n \pi y}{L}\right) \\
& p_{f}=\Delta x+\sum_{n=0}^{\infty} b_{n} \cos \left(\frac{n \pi x}{L}\right) \cosh \left(\frac{n \pi y}{L}\right) \\
& \psi_{f}=\Gamma x+\sum_{n=0}^{\infty} d_{n} \cos \left(\frac{n \pi x}{L}\right) \cosh \left(\frac{n \pi y}{L}\right)
\end{aligned}
$$

The constants $\Delta$ and $\Gamma$, which are the macroscopic gradients along the $x$-direction in the fractured zone, are related to the local fluxes $V_{0}$ and $J_{0}$ by

$$
\begin{aligned}
& V_{0}=-\alpha_{f} \Gamma-\frac{K_{f}}{\mu} \Delta \\
& J_{0}=-\sigma_{f} \Gamma-\alpha_{f} \Delta
\end{aligned}
$$

These relations can be easily inverted in order to obtain $\Delta$ and $\Gamma$ as functions of $V_{0}$ and $J_{0}$.

The form of this solution automatically satisfies the boundary conditions (4) and (5) at the upper and lower boundaries. The four unknown coefficients $a_{n}, b_{n}, c_{n}$ and $d_{n}$ are easily obtained by expressing the boundary conditions (3) at $|y|=h$. However, the series are often slowly convergent and some care has to be taken to obtain precise results.

Once these coefficients are obtained, the magnetic field $\boldsymbol{B}(\boldsymbol{R})$ at point $\mathrm{M}$ ( $c f$ Figure 1$)$ is obtained by using the Biot-Savart law

$$
\boldsymbol{B}(\boldsymbol{R})=\frac{\mu_{0}}{4 \pi} \iiint_{x^{\prime} \geq 0} I\left(\boldsymbol{R}^{\prime}\right) \times \frac{\boldsymbol{R}-\boldsymbol{R}^{\prime}}{\left|\boldsymbol{R}-\boldsymbol{R}^{\prime}\right|^{3}} \mathrm{~d} \boldsymbol{R}^{\prime}
$$

where $\mu_{0}$ is the magnetic permittivity $\left(=4 \pi 10^{-7}\right)$. The integration is performed over the whole medium (i.e., for $\left.x^{\prime} \geq 0\right) ; x^{\prime}$ is the abscissa of the point $M^{\prime} . B(R)$ has a single horizontal component $B_{z}$. The observation point $M$ is usually located slightly above the ground level (here, calculations are made for $1 \mathrm{~m}$ ). The integration over $z^{\prime}$ can be performed analytically because of the translational symmetry along the $z$-axis; the domain can also be reduced to the quarter plane $x \geq 0, y \geq 0, z=0$ because of the symmetry with respect to the $x$-axis.

Table 1. Range of studied parameters. All quantities are in SI units: $\sigma(\mathrm{S}), \alpha\left(\mathrm{m}^{2} \mathrm{~s}^{-1} \mathrm{~V}^{-1}\right), K\left(\mathrm{~m}^{2}\right)$. Data are for $J_{0}=0$.

\begin{tabular}{ccccccc}
\hline Case & $\sigma_{p}$ & $\alpha_{p}$ & $K_{p}$ & $\sigma_{f}$ & $\alpha_{f}$ & $K_{f}$ \\
\hline c1 & 0.01 & $310^{-9}$ & $10^{-12}$ & 0.1 & $310^{-9}$ & $10^{-10}$ \\
c2 & 0.1 & $310^{-9}$ & $10^{-10}$ & 0.1 & $310^{-9}$ & $10^{-10}$ \\
c3 & 0.001 & $310^{-9}$ & $10^{-12}$ & 0.01 & $310^{-9}$ & $10^{-10}$ \\
c4 & 0.01 & $10^{-8}$ & $10^{-12}$ & 0.1 & $10^{-8}$ & $10^{-10}$ \\
c5 & 0.01 & $310^{-9}$ & $10^{-11}$ & 0.1 & $310^{-9}$ & $10^{-9}$
\end{tabular}



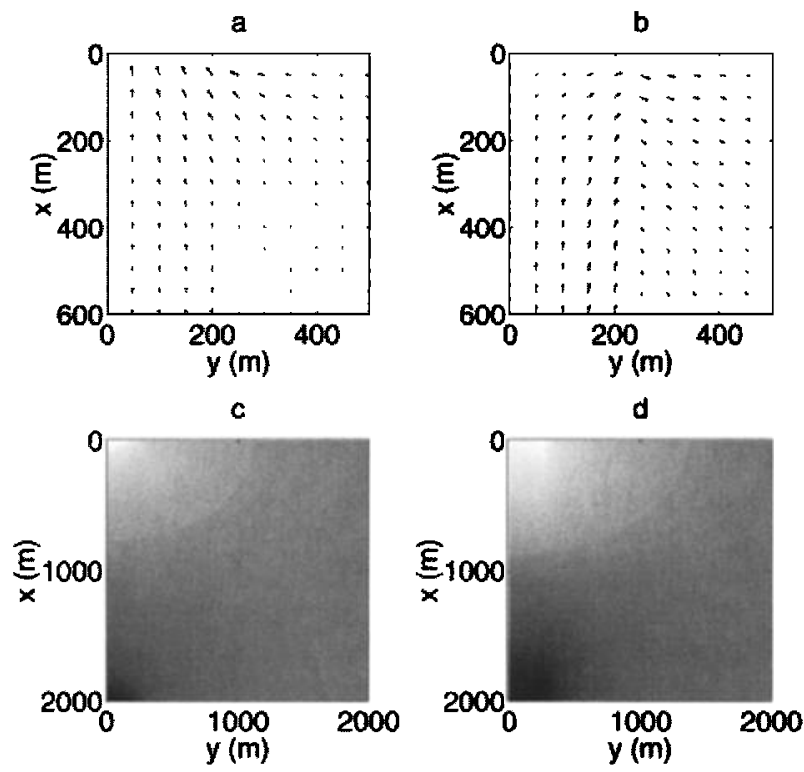

$\psi$

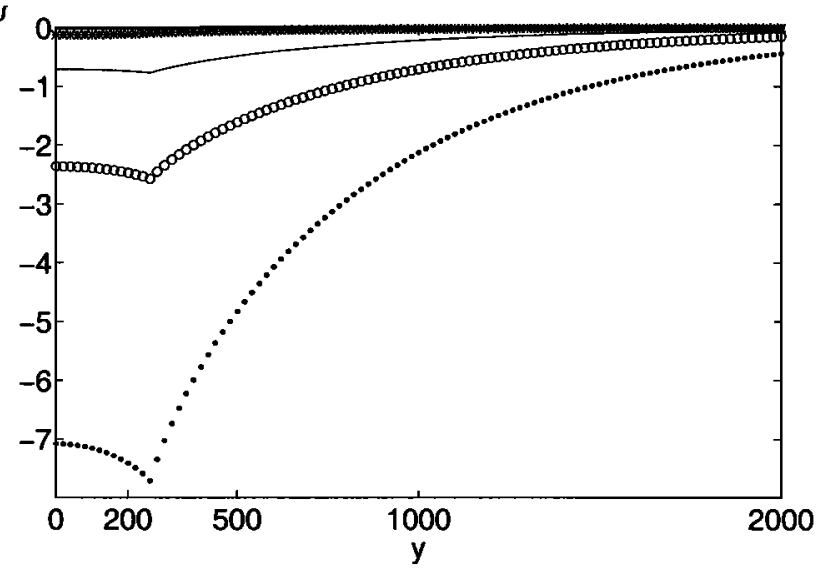

Figure 3. The potential field (Volts) on the ground $(x=0)$ as a function of the distance $y(\mathrm{~m})$ to the symmetry axis. Data are for: $c 1$ (solid line), $c 2\left({ }^{*}\right), c 3(\cdots), c 4(0), c 5(-\cdot)$. $c 5$ is overprinted by $c 1$.

Figure 2. The electromagnetic fields: the current densities ( $a$ and $b$ ) in the central upper part of the volcano; the potential fields $\psi$ (c and d) in the right half of the volcano. Data are for: case $c 2$ ( $a$ and c); case $c 1$ (b and d). Grey levels and arrow lengths are not consistent from one plot to another. The current densities in a and b at $x=y=200 \mathrm{~m}$ are of the order of $10^{-20}$ and $10^{-4} \mathrm{~A} / \mathrm{m}^{2}$, respectively. The potential differences in $\mathrm{c}$ and $\mathrm{d}$ between the dark and the white levels are equal to 2.7 and 1.5 Volts, respectively.

The many parameters which govern this situation can be divided into several groups. The first group is the geometry of the layers; since it is imposed by the geometry of the volcano, it is kept fixed

$$
h=260 m, \quad L=2000 m
$$

The second group includes the ingoing (and thus outgoing) fluxes. Usually, $J_{0}$ is taken equal to zero. An order of magnitude of the seepage velocity is estimated by assuming that when water is provided to the system, it falls under its own weight in the fractured zone

$$
V_{0}=\rho g \frac{K_{f}}{\mu}
$$

The third group of parameters consists of the local properties $K_{\imath}, \alpha_{i}$ and $\sigma_{\imath}$. For a given medium and fluid, they can be either measured or calculated given certain assumptions [Coelho et al., 1996]. Some of their properties are not intrinsic and also depend on water salinity and other physicochemical conditions. Hence, all these quantities can vary independently, at least to a first approximation.

The studied parameters are summarized in Table 1. The problem stated in Section 2 is rendered difficult by the large number of unknowns. With present knowledge, it was found preferable to make a sensitivity study around a set of central values, given as case $c 1$.

\section{Results and discussion}

Overall views of the electric fields are given in Figure 2 for the standard case $c 1$ and the homogeneous case $c 2$. For

the sake of clarity, only half of the central upper part is shown in Figure 2a and b. Because of the continuity of the fields at $|y|=h$, the fractured zone does not present any exceptional feature. These two representations were also used in order to check the solutions, their symmetries and the boundary conditions.

The electromagnetic fields predicted for ground level are displayed in Figures 3 and 4 for all the cases listed in Table 1. First of all, for case $c 1$, it should be emphasized that the order of magnitude of $\psi(\approx-1 \mathrm{~V})$ is consistent with the observations made by Michel and Zlotnick $[1998]$. However, the amplitude of $B_{z}(\approx 1 \mathrm{nT})$ is too small by a factor 10 [Zlotnicki and Le Mouel, 1990], a feature which will be discussed again later. These fields present a remarkable angular point at $|y|=h$ which is the limit of the fractured zone; this is consistent with the boundary conditions (3) where the normal components of the fluxes are continuous, but not the derivatives of the fields. Note that $\psi$ tends to zero far from the fractured zone. For $|y|<1000 \mathrm{~m}, B_{z}$ does not depend

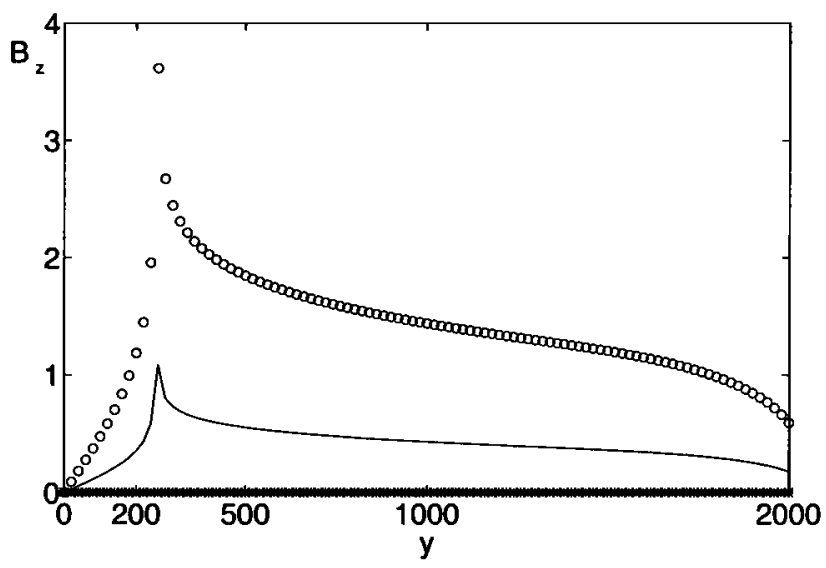

Figure 4. The magnetic field $B_{z}(\mathrm{nT})$ at $1 \mathrm{~m}$ above the ground as a function of the distance $y(\mathrm{~m})$ to the symmetry axis. Data are for: $c 1$ (solid line), $c 2\left(^{*}\right), c 3(\cdots), c 4(0), c 5$ $(-\cdot) . c 3$ and $c 5$ are overprinted by $c 1$. 
on the size of the integration domain of (12) along the $y$ axis; computations were performed for sizes equal to 4000 and $10000 \mathrm{~m}$ without any significant influence.

The influence of the heterogeneous character of the medium is demonstrated in these Figures by the homogeneous situation $c 2$ where the permeability of the porous medium is equal to that of the fractured zone. First, the kinks disappear from the curves as they should; second, $\psi$ and $B_{z}$ are reduced by a factor 10 . Hence, as is well known, the heterogeneous character of the underground medium is an essential feature to produce large fields. The residual fields which remain in $c 2$ are due to the no flux condition 4 at the surface of the porous medium.

The influence of the conductivities was analyzed in case $c 3$ where they were both divided by a factor 10 ( $c f$ Table 1 ). This parameter may vary widely; it depends on the local structure of the media and of the chemical content of the water. It is seen in Figures 3 and 4 that $\psi$ is multiplied by a factor 10 , while $B_{z}$ is superposed with case $c 1$ and thus does not appear clearly in Figure 4. Local water measurements are necessary to fix this important parameter.

The influence of larger coupling factors $\alpha_{2}$ was also clearly demonstrated by case $c 4$ where these two factors are multiplied by a factor 3 with respect to $c 1$. $\psi$ and $B_{z}$ are both roughly multiplied by a factor 3 . These orders of magnitude are the ones which fit the best the measured values of Zlotnıck and Le Mouel [1990]; note that the proposed variation is very small when compared with variations measured on plugs [Ishido and Mizutanı, 1981; Hovhannissian, 1999]. Hence, this factor should also be measured on samples extracted at La Fournaise.

Stronger permeabilities of the medium were studied in case $c 5$ where both permeabilities were multiplied by a factor 10 with respect to $c 1$. $u$ is modified accordingly and its influence on $\psi$ and $B_{z}$ is negligible.

Finally, the influence of an hypothesized outgoing current density $J_{0}$ was studied. It was shown that for $\left|J_{0}\right| \leq 10^{-7}$ $\mathrm{A} / \mathrm{m}^{2}$, the influence of this overall current is negligible on the external measurements $\psi$ and $B_{z}$; such a value is much larger than any likely atmospheric current density.

\section{Concluding remarks}

Magnetic and self-potential observations on the volcano [Zlotnicki and Le Mouel, 1990; Zlotnicki et al., 1993; Michel and Zlotnick, 1998] consist of the time dependent differences between the intensities of the geomagnetic field measured at various points distant of a few $\mathrm{km}$. Only variations are useful, since constant differences are generated by the magnetized rocks of the volcano. These variations have a typical amplitude of 5-10 $\mathrm{nT}$ and time constants ranging from a few minutes to weeks and years. They were conjectured to be due to the modulation of a fluid flow in the rift zone by forcing mechanisms such as volcano activity and associated changes in the stress field, atmospheric forcing (temperature, rain), and long term variations in the structure of the rift zone. This hypothesis is supported by the present steady-state analysis which yields the correct orders of magnitude.

This study also demonstrates the importance of the heterogeneous character of the underground medium. Moreover, it is crucial that properties such as conductivities and coupling coefficients are measured on plugs extracted from the volcano with the local water.
This study is preliminary; other boundary conditions could have been chosen which would modify the fields, but probably not the orders of magnitude which are obtained. Flow conditions could also have been varied, and storage effects considered. Thus, this study will be extended in a near future to a time-dependent analysis in a more realistic three-dimensional representation of the volcano.

Acknowledgments. This work was partly supported by a PNRN grant (CNRS/INSU).

\section{References}

Antraygues P. and M. Aubert, Self potential generated by twophase flow in a porous medium: experimental study and volcanological applications, $J$. Geophys. Res., B98, 22,27322,281, 1993.

Coelho D., Shapiro M., Thovert J.-F. and P.M. Adler, Electroosmotic phenomena in porous media, J. Collond Interf. Sci., $181,169-190,1996$.

de Groot S.R. and P. Mazur, Non-equilibrum thermodynamics, 510 pages, North-Holland, Amsterdam, 1969.

Fitterman D.V., Electrokinetic and magnetic anomalies associated with dilatant regions in a layered earth, $J$. Geophys. Res., 83, 5923-5928, 1978.

Fitterman D.V., Calculations of self-potential anomalies near vertical contacts, Geophys., 44, 195-205, 1979.

Hovhannissian G.M., IPGP, unpublished data, 1999.

Ishido T. and H. Mizutani, Experimental and theoretical basis of electrokinetic phenomena in rock-water systems and its applications to geophysics, $J$. Geophys. Res., 86, 1763-1775, 1981.

Johnston M.J.S., Review of electric and magnetic fields accompanying seismic and volcanic activity, Surveys in Geophys., 18 , 441-475, 1997.

Lorne B., Perrier F. and J.-P. Avouac, Streaming potential measurements, I: Properties of the electrical double layer from crushed rock samples, J. Geophys. Res., in press.

Martin R.J., Haupt R.W. and R.J. Greenfield, The effect of fluid flow on the magnetic field in low porosity crystalline rock, Geophys. Res. Lett., 12, 1301-1304, 1982.

Michel S. and J. Zlotnicki, Self-potential and magnetic surveying of La Fournaise volcano (Réunion Island): Correlations with faulting, fuid circulation, and eruption, J. Geophys. Res., B103, 17,845-17,857, 1998.

Mizutani H. and T. Ishido, A new interpretation of magnetic field variation associated with the Matsushiro earthquakes, $J$. Geomag. Geoelectr., 28, 179-188, 1976.

Morgan F.D., Williams E.R. and T.R. Madden, Streaming potential properties of Westerly granite with applications, J. Geophys. Res., B94, 12,449-12,461, 1989.

Nourbehecht B., Irreversible thermodynamic effects in inhomogeneous media and therr applacations in certain geological problems, Ph. D. thesis, Mass. Inst. Technol., Cambridge, MA, 1963.

Zlotnicki J. and J.-L. Le Mouel, Possible electrokinetic origin of large magnetic variations at La. Fournaise volcano, Nature, $343,633-635,1990$.

Zlotnicki J., Le Mouel J.-L., Delmond J.-C., Pambrun C. and H. Delorme, Magnetic variations on Piton de la Fournaise volcano and volcanomagnetic signals associated with the November 6 and 30, 1987, eruptions, J. Volcanol. Geotherm. Res., 56, 281$296,1993$.

P.M.Adler, J.-L. Le Mouel, Institut de Physique du Globe de Paris, 4, Place Jussieu, 75252-Paris Cedex 05, France, (e-mail: adler@io.cnusc.fr, lemouel@ipgp.jussieu.fr)

J. Zlotnicki, Laboratoire de Géophysique d'Orléans, 3D Avenue de la Recherche Scientifique, 47071 Orléans Cedex 02, France, (e-mail: jacques.zlotnicki@cnrs-orleans.fr)

(Received October 29, 1998; revised December 14, 1998; accepted January 27,1999 .) 